\title{
Editorial
}

\section{Nonlinear Functional Difference Equations with Applications}

\author{
Hua Su, ${ }^{1}$ Yuriy V. Rogovchenko, ${ }^{2}$ Youssef Raffoul, ${ }^{3}$ Yanbin Sang, ${ }^{4}$ and Fuyi Xu ${ }^{5}$ \\ ${ }^{1}$ School of Mathematics and Quantitative Economics, Shandong University of Finance and Economics, Jinan 250014, China \\ ${ }^{2}$ University of Agder, Kristiansand, Norway \\ ${ }^{3}$ Department of Mathematics, University of Dayton, Dayton, OH 45469-2316, USA \\ ${ }^{4}$ Department of Mathematics, North University of China, Taiyuan 030051, China \\ ${ }^{5}$ School of Science, Shandong University of Technology, Zibo 255049, China \\ Correspondence should be addressed to Hua Su; jnsuhua@163.com
}

Received 20 May 2013; Accepted 20 May 2013

Copyright (C) 2013 Hua Su et al. This is an open access article distributed under the Creative Commons Attribution License, which permits unrestricted use, distribution, and reproduction in any medium, provided the original work is properly cited.

It was a pleasure being responsible for the coordination of several proposals for research articles focusing on the theme nonlinear functional difference equations with applications. Studying the papers, we were happy to see that many researchers tend to focus on the study of the problems that we have been searching for years.

We appreciated the depth of ideas and the intention to give them a scientific approach. Therefore, we considered that 17 of the articles have to be considered in wider mathematical circles, being published in this journal in a special volume. It remains to be revealed in further comments, that will undoubtedly appear, how do they bring more knowledge.

We open the opportunity for the journal readers to make comments, that perhaps we were not able to put out, in relation to the following presentations.

In the paper titled "On the nonhomogeneous fourth-order p-Laplacian generalized Sturm-Liouville nonlocal boundary value problems" the authors study the nonlinear nonhomogeneous n-point generalized Sturm-Liouville fourth-order pLaplacian boundary value problem by using Leray-Schauder nonlinear alternative and Leggett-Williams fixed-point theorem.

The paper titled "Chaos in a discrete delay population model" is concerned with chaos in a discrete delay population model. The map of the model is proved to be chaotic in the sense of both Devaney and Li-Yorke under some conditions, by employing the snap-back repeller theory. Some computer simulations are provided to visualize the theoretical result.

The paper titled "A posteriori error estimates for a semidiscrete parabolic integrodifferential control on multimeshes" extends the existing techniques to study semidiscrete adaptive finite element approximation schemes for a constrained optimal control problem governed by parabolic integrodifferential equations. They first prove the uniqueness and existence of the solution of this optimal control problem. And then they derive the upper a posteriori error estimators for both the state and the control approximation, which are useful indicators in adaptive multimesh finite element approximation schemes.

The paper titled "On the expected discounted penalty function for a Markov regime-switching insurance risk model with stochastic premium income" proposed a Markovian regimeswitching risk model (also called Markov-modulated risk model) with stochastic premium income, in which the premium income and the claim occurrence are driven by Markovian regime-switching process. The purpose of this paper is to study the integral equations satisfied by the expected discounted penalty function. In particular, the discount interest force process is also regulated by Markovian regimeswitching process. Applications of the integral equations are given to be Laplace transform of the time of ruin, the deficit at ruin, and the surplus immediately before ruin occurs. For exponential distribution, the explicit expressions for these quantities are obtained. Finally, numerical example is also given to illustrate the effect of the related parameters on these quantities.

In the paper titled "Global regularity criterion for the magneto-micropolar fluid equations" the authors are concerned with the magneto-micropolar fluid equations in 
R3. Using Littlewood-Paley decomposition, they obtain an Osgood-type global regularity criterion for the system.

In the paper titled "Eigenvalue of fractional differential equations with p-Laplacian operator," by constructing upper and lower solutions, the authors obtained the existence of positive solutions for the fractional order eigenvalue problem with p-Laplacian operator. The authors results can be applied to the forefront of research of real estate asset securitization.

In the paper titled "Generalized antiperiodic boundary value problems for the fractional differential equation with $p$ Laplacian operator" the existence of solutions about generalized antiperiodic boundary value problems for the fractional differential equation with p-Laplacian operator is obtained under some suitable hypotheses. The author proof is based on fixed point theorem and contraction mapping principle. Furthermore, three examples are also given to illustrate the results.

In the paper titled "Studies on a double Poisson-Geometric insurance risk model with interference" the authors mainly study a generalized double Poisson-Geometric insurance risk model. By martingale and stopping time approach, they obtain adjustment coefficient equation, the Lundberg inequality, and the formula for the ruin probability. Also the Laplace transformation of the time when the surplus reaches a given level for the first time is discussed, and the expectation and its variance are obtained. Finally, we give the numerical examples.

In the paper titled "Finite unions of D-spaces and applications of nearly good relation" the authors give some results on finite unions of D-spaces. It is proved that if a space is the union of finitely many locally compact D-subspaces, then it is a D-space. It follows that a space is a D-space if it is the union of finitely many locally compact submetacompact subspaces. And a space is a D-space if it is the union of a D-subspace with a locally compact D-subspace. At last, some examples are given to exhibit the applications of nearly good relation to discover D-classes.

In the paper titled "The solutions of mixed monotone Fredholm-type integral equations in Banach spaces," by means of the monotone iterative techniques without any compactness conditions, the author obtains the iterative unique solution of nonlinear mixed monotone Fredholm-type integral equations in Banach spaces. They results are even new to convex and concave quasi operator. And then the author applies these results to the two-point boundary value problem of second-order nonlinear ordinary differential equations in ordered Banach spaces.

In the paper titled " $A$ regularity criterion for the magnetomicropolar fluid equations in $\dot{B}_{\infty, \infty}^{-1}$ " the authors study the Cauchy problem for the magneto-micropolar fluid equations in three dimensions spaces. A new logarithmically improved regularity criterion for the magneto-micropolar fluid equations is established in terms of the pressure in the homogeneous Besov space.

In the paper titled "Stabilization of discrete-time planar switched linear systems with impulse" the authors study the stabilization problem of discrete-time planar switched linear systems with impulse. When all subsystems are controllable, based on an explicit estimation on the state transition matrix, they establish a sufficient condition such that the switched impulsive system is stabilizable under arbitrary switching signal with given switching frequency. When there exists at least one uncontrollable subsystem, a sufficient condition is also given to guarantee the stabilization of the switched impulsive system under appropriate switching signal.

In the paper titled "Characterizations of strongly paracompact spaces" the author proves that a countably paracompact normal space (a perfectly normal space or a monotonically normal space) is strongly paracompact if and only if every increasing open cover of the space has a star-countable open refinement. Moreover, it is shown that a space is linearly D, provided that every increasing open cover of the space has a point-countable open refinement.

In the paper titled "Positive solutions for three-point boundary value problem of fractional differential equation with p-Laplacian operator" the authors investigate the existence of multiple positive solutions for three-point boundary value problem of fractional differential equation with p-Laplacian operator. By applying monotone iterative technique, some sufficient conditions for the existence of multiple positive solutions are established; moreover iterative schemes for approximating these solutions are also obtained, which start off a known simple linear function. In the end, an example is worked out to illustrate the main results.

In the paper titled "Blow-up criteria for three-dimensional Boussinesq equations in Triebel-Lizorkin spaces" the author establishes a new blow-up criteria for solution of the threedimensional Boussinesq equations in Triebel-Lizorkin spaces by using Littlewood-Paley decomposition.

In the paper titled "Bifurcation analysis in a delayed diffusive Leslie-Gower model" the authors deal with the stability analysis of the equilibria of the model and show the existence of Hopf bifurcation at the positive equilibrium under some conditions. Furthermore, they investigate the stability and direction of bifurcating periodic orbits by using normal form theorem and the center manifold theorem.

In the paper titled "Existence and uniqueness of positive solutions to nonlinear second order impulsive differential equations with concave or convex nonlinearities," by using a new fixed point theorem of generalized concave operators, the authors present criteria which guarantee the existence and uniqueness of positive solutions to nonlinear two-point boundary value problems for second-order impulsive differential equations with concave or convex nonlinearities.

\section{Acknowledgments}

We would like to express appreciation to the authors for their excellent contributions and patience in assisting us. Finally, the fundamental work of all reviewers on these papers is also very greatly acknowledged.

Hua Su

Yuriy V. Rogovchenko Youssef Raffoul Yanbin Sang Fuyi Xu 


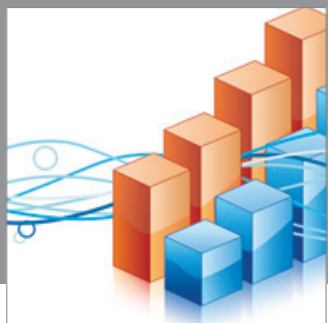

Advances in

Operations Research

mansans

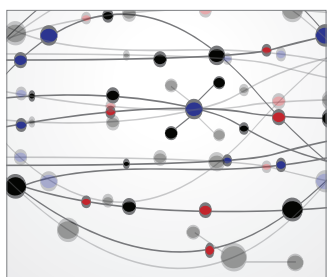

The Scientific World Journal
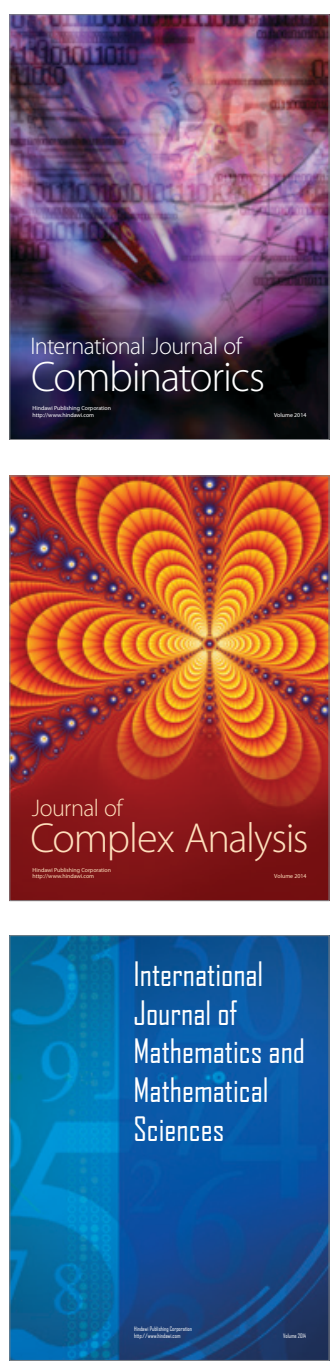
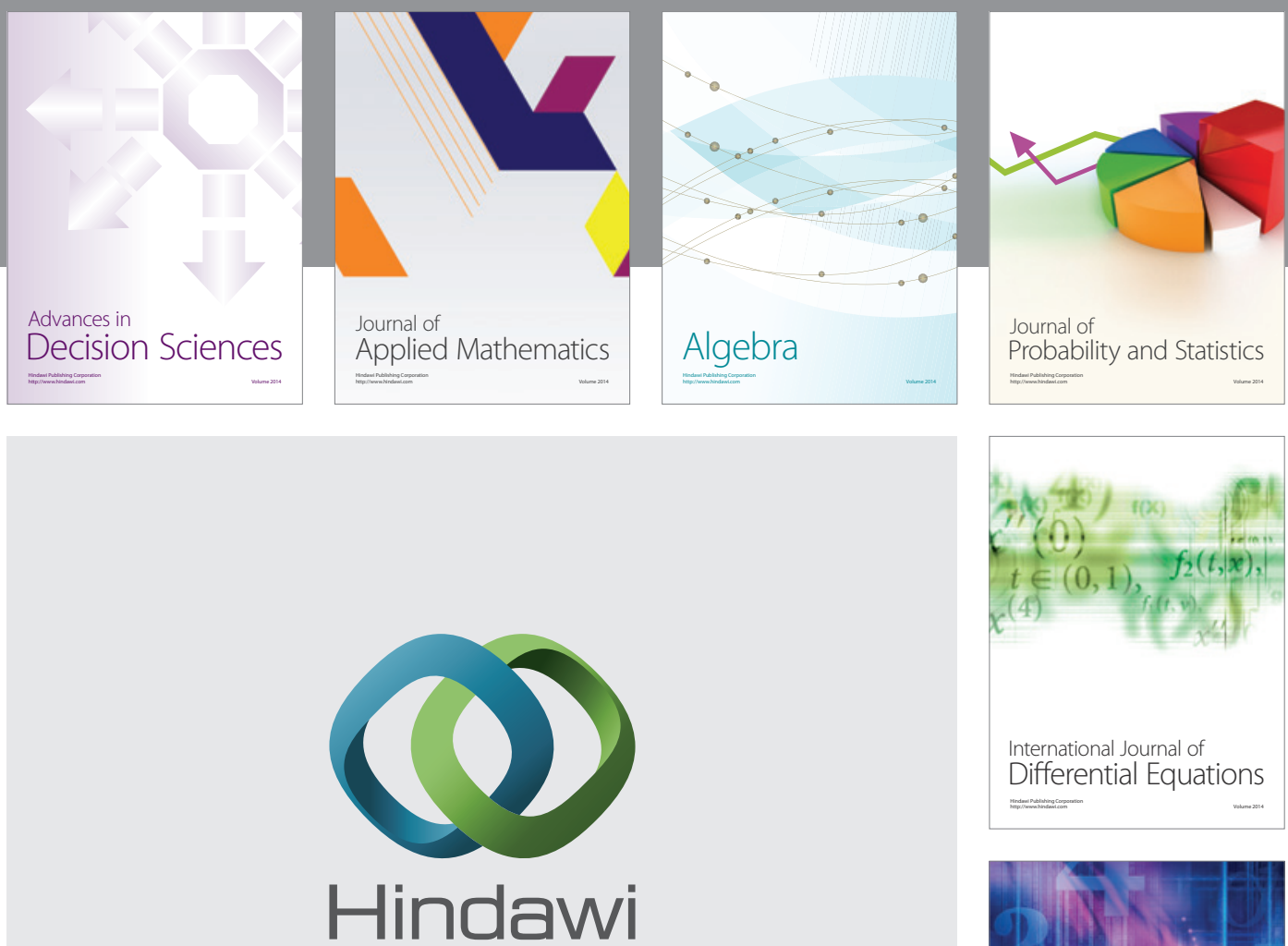

Submit your manuscripts at http://www.hindawi.com
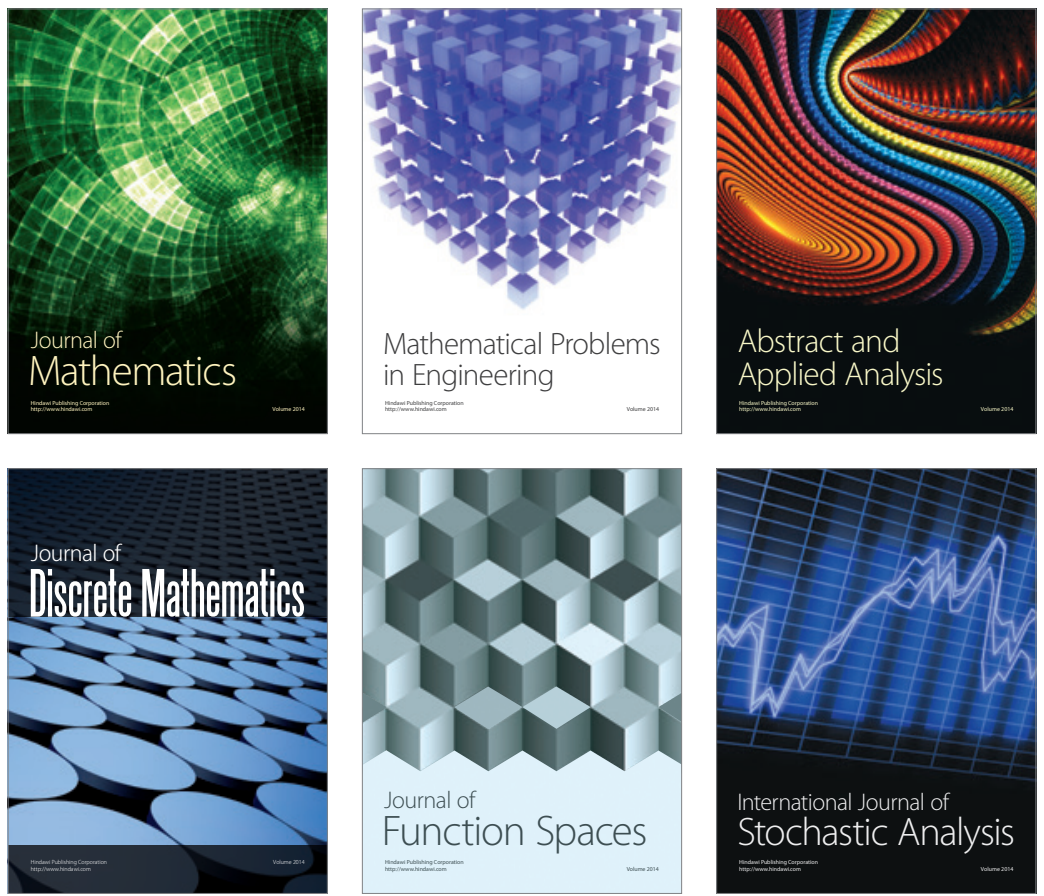

Journal of

Function Spaces

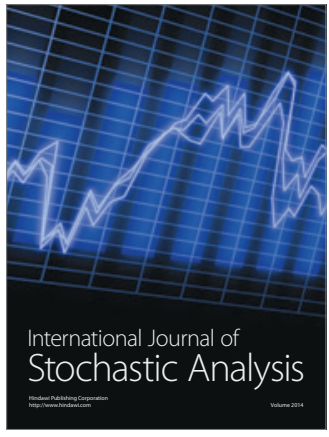

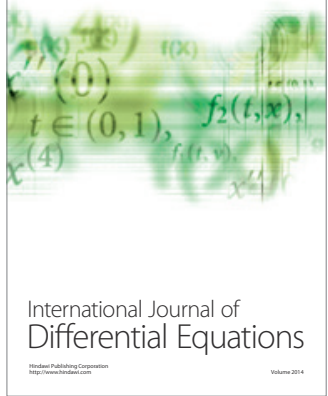
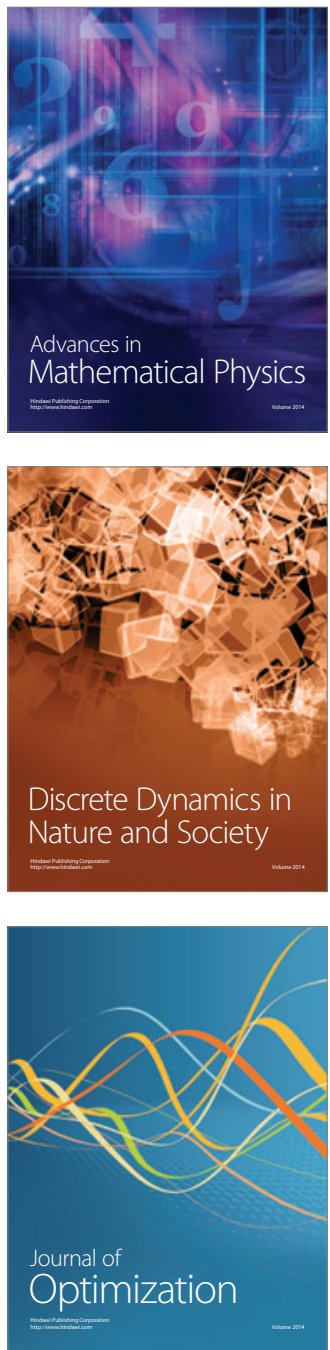